\title{
Mechanism of Atorvastatin in Improving Cardiac Function in a Rat Model of Myocardial Infarction
}

\author{
YINGYING FU 1 , SHUNDA WANG ${ }^{2}$ AND QIANWEI CUI ${ }^{3 *}$ \\ ${ }^{1}$ Department of Emergency, ${ }^{2}$ Department of Rehabilitation Medicine, 3Department of Cardiovascular, Shaanxi Provincial Peo- \\ ple's Hospital, Xi'an, 710068, Shaanxi, China
}

Fu et al.: Atorvastatin Improved Cardiac Function after Myocardial Infarction

\begin{abstract}
In order to explore the mechanism of atorvastatin on cardiac function after myocardial infarction in rats, 40 male Sprague Dawley rats were divided into 4 groups. There were 8 rats in blank group, 8 in control group, 8 in sham operated group and 16 in atorvastatin group. Atorvastatin group is divided into high dose group (30 mg/kg/d) and low dose group $(10 \mathrm{mg} / \mathrm{kg} / \mathrm{d}), 8$ rats in each group. A rat model of myocardial infarction was established by ligating the left anterior descending artery. The effect of atorvastatin on cardiac function after myocardial infarction was observed. Cardiac function of rats that included the left ventricular ejection fraction, left ventricular end systolic diameter, left ventricular end diastolic diameter and left ventricular fraction shortening were measured and analysed using ultrasound. Blood lipids of rats in each group including total cholesterol, triglycerides, high density lipoprotein and low-density lipoprotein were measured. After $4 \mathrm{w}$ of treatment rats were sacrificed, myocardial tissue was stained with $\mathrm{HE}$ and Masson to observe cardiac fibrosis. Results showed that $4 \mathrm{w}$ after ligation, compared to the control group, the levels of left ventricular end systolic and left ventricular end diastolic diameter was decreased significantly in atorvastatin-treated groups $\mathrm{A1}$ and A2, while left ventricular ejection fraction and left ventricular fraction shortening increased significantly. On $4 \mathrm{w}$ after ligation, total cholesterol and lowdensity lipoprotein cholesterol levels in $\mathrm{A1}$ and $\mathrm{A2}$ groups were significantly lower than those in control group. Compared to the control group, the areas of myocardial infarction and cardiac fibrosis in $\mathrm{A} 1$ and A2 groups were significantly reduced. It is concluded that atorvastatin could inhibit apoptosis of myocardial cells, reverse ventricular remodeling and improve cardiac function after myocardial infarction, and these findings are of clinical significance.
\end{abstract}

Key words: Atorvastatin, myocardial infarction, cardiac function, ventricular remodeling

In recent years, myocardial infarction (MI) has become a common disease that seriously threatens the health of all human beings. The mortality rate in the world remains high for a long time, and about $30 \%$ of the patients with MI will eventually develop into heart failure ${ }^{[1]}$. Heart failure is a group of pathophysiological syndromes with various causes of cardiac insufficiency. The main characteristics are left ventricular and/or right ventricular dysfunction and changes in neurohumoral regulation, which seriously affect the quality of life of patients and even lead to death ${ }^{[2]}$. More recntly, the incidence of metabolic diseases such as diabetes, hypertension and hyperlipidemia caused by smoking and obesity is increasing year by year, and the incidence of MI is getting higher and higher ${ }^{[3]}$. Studies showed that ventricular remodeling occurs within hours of the onset of MI, so as to compensate for myocardial tissue loss caused by MI. At this time, the structure of the ventricle has changed, and progressive expansion and shape changes have taken place, which may lead to arrhythmia, heart failure and heart rupture ${ }^{[4]}$. Therefore, inhibiting and blocking the occurrence and development of ventricular remodeling is the main way to prevent and treat heart failure after MI, and also the key to improve cardiac function. Although percutaneous coronary intervention (PCI) is the most effective treatment for MI with ischemia-reperfusion, there is still about $7 \%$ mortality in the near future ${ }^{[5]}$. Therefore, drug treatment is still an indispensable intervention in the treatment of MI.

At present, the drugs used to treat MI mainly include nitrates, statins, angiotensin converting enzyme inhibitors, angiotensin receptor blockers and so on.

*Address for correspondence

E-mail: cuiqianweiqw2012@126.com 
In the previous studies, it is found that statins can not only regulate blood lipids, but also play a certain role as antiinflammatory, antioxidative stress, inhibiting apoptosis, protecting myocardium and other aspects. These can effectively reduce the probability of cardiovascular events, so they are also widely used in primary prevention and secondary prevention of coronary heart disease ${ }^{[6]}$. Based on this, a rat model of MI was constructed to further explore the mechanism and application value of atorvastatin in delaying ventricular remodeling, improving myocardial function of patients with MI, preventing and treating coronary heart disease, so as to provide a basis for clinical medication $^{[7,8]}$.

\section{MATERIALS AND METHODS:}

Forty male Sprague Dawley (SD) rats, $(182.11 \pm 19.25 \mathrm{~g})$ aged 6-8 w were selected. Under normal environment, rats were fed with granular diet and clean drinking water for $1 \mathrm{w}$, and maintained under constant temperature and humidity $\left(22-24^{\circ}, 50-60 \%\right)$. All rats were then fed with a high-fat diet containing $3.8 \%$ cholesterol, $11 \%$ lard, $0.2 \%$ methylthiouracil and $85 \%$ common feed for $4 \mathrm{w}$. The rats were divided into 4 groups, blank group (8 rats), control group (8 rats), sham-operated group ( 8 rats) and atorvastatin-treated group (16 rats). The atorvastatin group was further divided into the high dose group (A1: $30 \mathrm{mg} / \mathrm{kg} / \mathrm{d}$ ) and low dose group (A2: $10 \mathrm{mg} / \mathrm{kg} / \mathrm{d}$ ), with 8 rats in each group.

The rats in the blank group were not given any treatment. In the sham-operated group, the left anterior descending coronary artery was not ligated. In the control group, the anterior descending branch of left coronary artery was ligated and $2 \mathrm{ml}$ saline was given every $24 \mathrm{~h}$ for $4 \mathrm{w}$. In group A1, the left anterior descending coronary artery of rats was ligated and atorvastatin $40 \mathrm{mg} / \mathrm{kg}$ was given every $24 \mathrm{~h}$ ( $2 \mathrm{ml}$ solution in saline) is given orally for 4. In group A2, the left anterior descending coronary artery was ligated and $10 \mathrm{mg} / \mathrm{kg}$ atorvastatin was given every $24 \mathrm{~h}$ ( $2 \mathrm{ml}$ solution in saline) for $4 \mathrm{w}$. During the experiment, all rats were maintained under the same environment in individual cages with free access to feed and drinking water. The experiments were carried out in strict accordance with the requirements of Standards for the Operation of Laboratory Animals.

\section{Construction of rat model of myocardial infarction:}

In this study, a rat model of myocardial infarction was established by coronary ligation. First, after $4 \mathrm{w}$ of feeding with high-fat diet, atherosclerosis rat model was established. After the last intragastric administration, feed and water were deprived for $12 \mathrm{~h}$ before operation. Rats were anesthetized by intraperitoneal injection of $10 \%$ chloral hydrate $(0.3 \mathrm{ml} / 100 \mathrm{~g})$, fixed on a rat operating table, the skin of the neck and chest was prepared for surgery. Endotracheal intubation was performed. A longitudinal incision of about $1.3 \mathrm{~cm}$ was made, muscular layer is bluntly separated with tweezers to expose the trachea and thyroid cartilage of rats. A trocar was inserted into the second tracheal cartilage ring of rat trachea at 45 -degree angle. When there is a sense of breakthrough, the needle was inserted horizontally for $2 \mathrm{~cm}$. The needle core is withdrawn and connected with the ventilator (Shenzhen Reward Life Science and Technology Co., Ltd; model: HX-101E). Thoracotomy was performed after connecting the rats with the ECG monitoring device and the chest is opened in the third and fourth rib space after the respiratory and ECG indices were stable. After cutting the skin layers the muscular layer was bluntly separated, the thorax was enlarged with a vascular forceps to expose the heart, the pericardium was torn open with ophthalmic forceps. A cotton ball moistened with normal saline was used to gently press the left lung of rats to expose the field of vision of the area to be ligated. The left anterior descending branch was located. Holding a needle in the right hand, the left anterior descending coronary artery was ligated with 6-0 suture at $1-2 \mathrm{~mm}$ below the junction of left atrium and pulmonary conus. The range is about $1 \mathrm{~mm}$ deep and $3 \mathrm{~mm}$ wide. If malignant arrhythmia occurred during the operation, the thoracic cavity was be temporarily clamped for chest compression.

The success criteria of the model construction were as follows. The color of heart tissue after ligation and the electrocardiogram, if the ligation area and apical myocardium became white, local myocardial movement was weakened and the electrocardiogram showed ST segment elevation it indicated that the myocardial infarction model was successfully constructed $^{[7,8]}$. After the successful establishment of the model, rats were sutured after the vital signs were found to be stable. Gentamicin $\left(2.4 \times 10^{4} / \mathrm{kg} / \mathrm{d}\right)$ was injected intraperitoneally for $3 \mathrm{~d}$ to prevent infection. After awakening, the rats were withdrawn from the ventilator and ECG monitoring and returned to the cage to continue feeding.

\section{Evaluation index and detection method:}

Before and $4 \mathrm{w}$ after the establishment of MI model in rats, the changes of cardiac function in each group were detected using color Doppler ultrasound diagnostic 
instrument (Shenzhen Mindray Biomedical Electronics Co., Ltd.). Rats are anaesthetized with $10 \%$ chloral hydrate and fixed on the rat table in the supine position. Three continuous, stable and complete cardiac cycles were measured by M-mode echocardiography after shaving the left anterior thoracic region hair. Relevant indicators were recorded. Measurements included the left ventricular ejection fraction (LVEF), left ventricular end systolic diameter systolic (LVESD), left ventricular end diastolic diameter (LVEDD) and left ventricular fraction shortening (LVFS), which were analysed using ultrasound software (BL-420 bioinformatics collection system).

Blood lipid determinations includes total cholesterol (TC), triglyceride (TG), high density lipoprotein (HDL-C) and low-density lipoprotein (LDL-C). TC and TG were detected by enzymatic method. HDL-C and LDL-C were detected by selective precipitation method. The specific procedures were strictly implemented according to the instructions of the manufacturer.

After the last ultrasound image acquisition, all rats were executed, and hearts removed after thoracotomy. In pre-cooled saline solution, the heart was quickly washed to remove blood and connective tissue. After $24 \mathrm{~h}$ of fixation with $10 \%$ neutral formalin, the apical tissue was removed and stained with HE. Paraffin was used to encapsulate tissues. After cooling, slices of $3 \mu \mathrm{m}$ thickness were cut, dewaxed in xylene for $15 \mathrm{~min}$, washed, dehydrated in 100, 95 and $85 \%$ alcohol for $3 \mathrm{~min}$, exposed to hematoxylin staining for 3-5 min and washed. The slices were separated in $1 \%$ alcoholic hydrochloric acid and after returning to blue in warm water ware stained with eosin. After dehydration in alcohol, the slices were soaked in xylene and sealed with neutral gum. Cell morphology and structure were observed under light microscope at $400 \mathrm{X}$ magnification. The remaining cardiac tissue was stained using Masson stain. Myocardial tissue specimens were fixed with $4 \%$ paraformaldehyde, embedded in paraffin, stained with hematoxylin for $3 \mathrm{~min}$, rinsed and the cells were differentiated by $1 \%$ hydrochloric acid alcohol for 3 to $5 \mathrm{~s}$. After rinsing, the cells returned to blue in warm water for $1 \mathrm{~min}$, rinsed again and were stained with acid fuchsine of ponceau for $3 \mathrm{~min}$, differentiated with $1 \%$ phosphomolybdic acid for $1 \mathrm{~min}$ and stained with $2 \%$ aniline blue for $1 \mathrm{~min}$. The specimens were dehydrated in $95 \%$ alcohol and sealed with neutral gum after drying. Under optical microscope, the proportion of cardiac fibrosis was analyzed by Image $\mathrm{J}$ software. The proportion of fibrosis is equal to the area of fibrosis/ cardiac area.

\section{Pharmacological effects of atorvastatin:}

Atorvastatin is a 3-hydroxy-3-methylglutaric acid monoacyl coenzyme A reductase inhibitor, which can inhibit the activity of HMG-CoA reductase, thereby inhibiting the synthesis of cholesterol in liver and lowering the level of total cholesterol in plasma. Atorvastatin can also increase LDL uptake and catabolism by increasing the number of LDL receptors on the surface of hepatocytes. In recent years, the non-lipid-lowering effect of statins in cardiovascular diseases has attracted more and more attention. Some foreign scholars have found that statins can also inhibit and reverse ventricular remodeling. The mechanism of statins in improving ventricular remodeling after myocardial infarction may include improving vascular endothelial function, antioxidant effect, improving insulin resistance, reducing the activity of angiotensin converting enzyme, thereby reducing the production of angiotensin II, and interfering with the immune response in the occurrence of myocardial infarction, which has the function of protecting the heart.

\section{Statistical methods:}

In this study, SPSS 20.0 statistical software was used for analysis and the measurement data were expressed as mean \pm standard deviation. Firstly, whether the samples conform to normal distribution is tested. If not, non-parametric test is carried out. If the results are consistent, one-way variance analysis is used and homogeneity test of variance is carried out. $\mathrm{P}<0.05$ has statistical significance.

\section{RESULTS AND DISCUSSION}

According to the data in Tables 1 and 2, the LVEDD and LVESD in the control group at $4 \mathrm{w}$ after operation were significantly higher than those before operation. LVEF and LVFS were significantly lower than those before operation $(\mathrm{P}<0.05)$. LVEDD and LVESD in group A2 were higher than those before operation, while the levels of LVEF and LVFS were lower than those before operation. Four weeks after operation, cardiac functional indices approached normal level, and the difference was statistically significant $(\mathrm{P}<0.05)$. The cardiac function indices in group A1 were similar to those in group A2. After operation, the levels of LVEDD and LVESD were higher than those before operation, while the levels of LVEF and LVFS were 
www.ijpsonline.com

TABLE 1: COMPARISON OF CARDIAC FUNCTION OF RATS IN EACH GROUP BEFORE LIGATION

\begin{tabular}{lcccc}
\hline Group & LVEDD $(\mathrm{cm})$ & LVESD $(\mathrm{cm})$ & LVEF $(\%)$ & LVFS $(\%)$ \\
\hline Blank group & $0.60 \pm 0.03$ & $0.33 \pm 0.04$ & $83.99 \pm 5.09$ & $51.13 \pm 4.56$ \\
Control group & $0.60 \pm 0.05$ & $0.36 \pm 0.05$ & $81.49 \pm 8.20$ & $52.03 \pm 3.94$ \\
Sham-operated group & $0.60 \pm 0.04$ & $0.34 \pm 0.04$ & $79.60 \pm 6.45$ & $53.70 \pm 5.02$ \\
A1 & $0.65 \pm 0.04$ & $0.38 \pm 0.09$ & $82.78 \pm 5.37$ & $52.34 \pm 3.77$ \\
A2 & $0.65 \pm 0.05$ & $0.39 \pm 0.07$ & $82.04 \pm 8.01$ & $53.16 \pm 4.55$ \\
\hline
\end{tabular}

COMPARISON OF CARDIAC FUNCTION OF RATS IN EACH GROUP BEFORE LIGATION Data is expressed as mean \pm SD, sample size is 8.

TABLE 2: COMPARISON OF CARDIAC FUNCTION OF RATS IN EACH GROUP 4 WEEKS AFTER LIGATION

\begin{tabular}{lcccc}
\hline Group & LVEDD $(\mathrm{cm})$ & LVESD $(\mathrm{cm})$ & LVEF (\%) & LVFS (\%) \\
\hline Blank group & $0.60 \pm 0.03$ & $0.33 \pm 0.05$ & $83.82 \pm 5.02$ & $51.07 \pm 4.83$ \\
Control group & $1.26 \pm 0.18^{*}$ & $0.80 \pm 0.13^{*}$ & $42.93 \pm 5.32^{*}$ & $24.05 \pm 5.22^{*}$ \\
Sham-operated group & $0.60 \pm 0.05$ & $0.35 \pm 0.07$ & $80.84 \pm 7.48$ & $53.04 \pm 4.24$ \\
A1 & $0.73 \pm 0.05^{*} \#$ & $0.53 \pm 0.09^{*} \#$ & $68.97 \pm 7.86^{*} \#$ & $48.00 \pm 5.43^{*} \#$ \\
A2 & $0.78 \pm 0.05^{*} \#$ & $0.52 \pm 0.10^{*} \#$ & $70.53 \pm 5.42^{*} \#$ & $48.44 \pm 4.58^{*} \#$ \\
\hline
\end{tabular}

Data is expressed as mean \pm SD, sample size is 8 , ${ }^{*}$ compared to the same group before operation, $P<0.05$; \#compared to the control group after operation, $\mathrm{P}<0.05$.

TABLE 3: COMPARISON OF BLOOD LIPIDS IN RATS OF EACH GROUP AFTER 4 WEEKS AFTER SURGERY

\begin{tabular}{lcccc}
\hline Group & TC & TG & HDL-C & LDL-C \\
\hline Blank group & $2.49 \pm 0.17$ & $1.35 \pm 0.14$ & $1.44 \pm 0.17$ & $0.70 \pm 0.15$ \\
Control group & $2.80 \pm 0.28$ & $1.35 \pm 0.18$ & $1.41 \pm 0.20$ & $0.70 \pm 0.13$ \\
Sham-operated group & $2.50 \pm 0.19$ & $1.44 \pm 0.14$ & $1.53 \pm 0.18$ & $0.65 \pm 0.17$ \\
A1 & $1.60 \pm 0.30^{*}$ & $1.35 \pm 0.17$ & $1.52 \pm 0.17$ & $0.40 \pm 0.12^{*}$ \\
A2 & $1.79 \pm 0.26^{*}$ & $1.34 \pm 0.17$ & $1.53 \pm 0.17$ & $0.44 \pm 0.12^{*}$ \\
\hline
\end{tabular}

Data is expressed as mean $\pm \mathrm{SD} \mathrm{mM}$, sample size is 8 , *compared with the control group, $\mathrm{P}<0.05$.

lower than those before operation $(\mathrm{P}<0.05)$. There is no significant difference between the blank group and the sham-operated group.

At $4 \mathrm{w}$ after operation, the levels of LVEDD and LVESD in groups A1 and A2 were significantly lower than those in the control group, while the levels of LVEF and LVFS were significantly higher than those in the control group $(\mathrm{P}<0.05)$. From echocardiography, it was observed that the peak systolic velocities of anterior septum, posterior septum, left ventricular anterior wall and left ventricular apex increased in groups A1 and A2, which further showed that atorvastatin could improve left ventricular systolic function after MI and inhibit the enlargement of cardiac cavity. It was found that the indices of cardiac function in rats treated with atorvastatin appeared to be stable $4 \mathrm{w}$ after operation, which could guide the treatment cycle of ventricular remodeling after MI. Continuous administration of atorvastatin could significantly improve the cardiac function of rats after MI.

According to the data in Table 3, there was no significant difference HDL-C and TG levels between the groups $4 \mathrm{w}$ after surgery. The TC level of A1 and A2 group rats were $1.60 \pm 0.30$ and $1.79 \pm 0.26 \mathrm{mM}$, respectively. LDL-C level was $0.40 \pm 0.81$ and $0.44 \pm 0.90 \mathrm{mM}$, respectively, which was significantly lower than that of the control group $(\mathrm{P}<0.05)$.

Studies have confirmed that the incidence of MI is positively correlated with the level of LDL-C in the blood and negatively correlated with the level of HDL-C. Therefore, rational regulation of blood lipids is of great significance for the prevention and treatment of MI and is also an important means to control cardiovascular risk factors. Atorvastatin is widely used in clinical practice as a common drug for regulating blood lipids. Its chemical structure is similar to 3-hydroxy-3methylglutaryl coenzyme A reductase (HMG-CoA). Its mechanism of action is to competitively inhibit the ratelimiting enzymes in the process of hepatocyte synthesis of cholesterol and reduce the synthesis of cholesterol. At the same time, atorvastatin can lower the levels of plasma LDL-C and VLDL-C by negative feedback regulation and raise the level of HDL-C.

From the myocardial HE staining of the rats in each group in fig. 1, it can be seen that the myocardial sections of the blank group have clear texture, complete capsule. It can be seen that the obvious cell transverse structure, the nucleus and the distribution of the patina are normal. There is no inflammatory cell infiltration or increased fibrous tissue. Myocardial cell lines are still visible in 


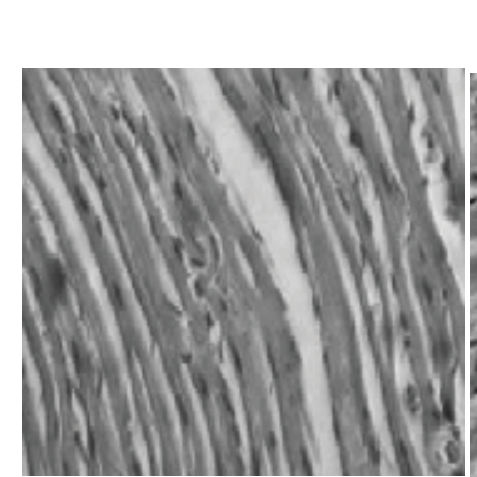

A

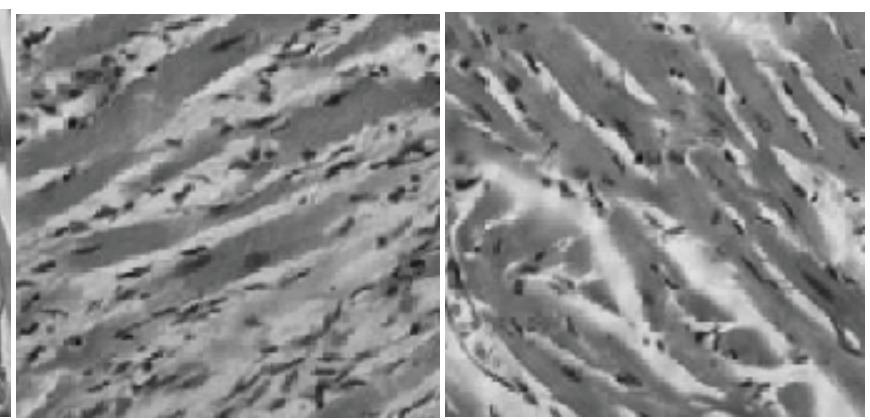

B

C

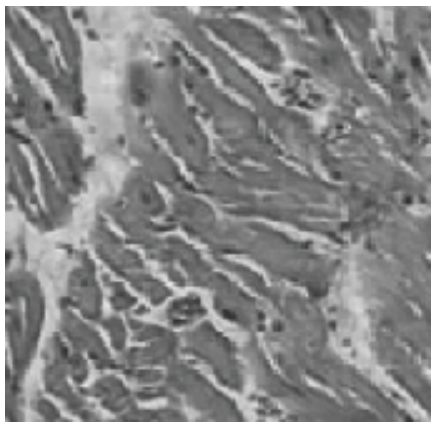

D

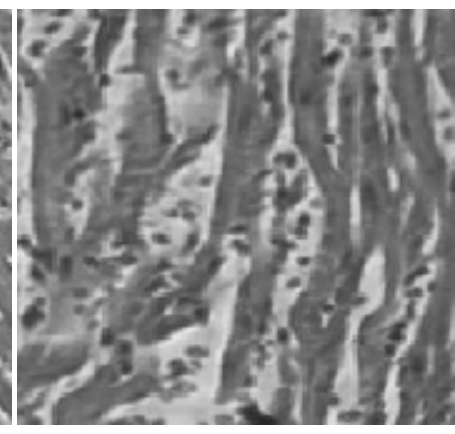

$\mathbf{E}$

Fig. 1: HE staining results of myocardium of rats in each group (400X)

A. Blank group, B. control group, C. sham-operated group, D. A1 group and E. A2 group

the sham-operated rats, and the cell structure is intact. There is a small amount of inflammatory cell infiltration and fibrous tissue hyperplasia. In the control group, the cells in the myocardial sections were scattered, the cell lines were not clear, the cell membrane structure was incomplete, and the cells were necrotic. Nuclear pyknosis and dissolution have emerged. Some muscle fibers in the remaining small amount of myocardial fibers were broken, and a large amount of inflammatory cell infiltration and fibrous tissue hyperplasia occur. In the A1 and A2 groups of rats, myocardial cells were found in the myocardial sections, the cell lines were clear, and the cell membrane structure can be seen. The necrosis of myocardial cells was significantly reduced compared to the control group, the infiltration of inflammatory cells was reduced, and the proliferation of fibrous tissue was significantly improved. Compared to the A2 group, the myocardial necrosis, inflammatory cell infiltration, and tissue fibrosis were much less in the A1 group, and the normal structure of the cardiomyocytes were more. The myocardial section of group A1 is better than that of group A2.

After MI, there will be a large number of inflammatory factors exudation and activation of fibroblasts, which can repair the heart tissue and cause the occurrence of ventricular remodeling. Therefore, in this study, the myocardial fibrosis after MI in rats of each group is further analyzed. The results of Masson staining (fig. 2) showed that the proportion of MI area and myocardial fibrosis in the blank group is 0 . Compared with the blank group, the proportion of fibrosis increases significantly in the control group $(\mathrm{P}<0.05)$. According to the formula, the proportion of $\mathrm{MI}$ area in control group, A1 group and A2 group were $0.32 \pm 0.05,0.20 \pm 0.09$ and $0.22 \pm 0.08$, respectively. A1 and A2 groups were compared with the control group, and the difference was significant $(\mathrm{P}<0.05)$. The proportions of cardiac fibrosis in control group, A1 group and A2 group were $0.20 \pm 0.02,0.09 \pm 0.02$ and $0.12 \pm 0.02$, respectively. A1 and A2 groups are compared with the control group, and the difference was significant $(\mathrm{P}<0.05)$. It can be concluded that atorvastatin significantly reduced the proportion of MI area and myocardial fibrosis in rats after MI, indicating that atorvastatin can inhibit myocardial fibrosis.

After MI, the mechanism of decreased myocardial contractility mainly includes changes in myocardial contraction-related proteins, disturbance of myocardial energy metabolism and abnormal excitation-contraction coupling. The more serious pathophysiological changes after MI were abnormal ventricular structure and function. Ventricular remodeling is a process of ventricular compensation, which can lead to a series of pathophysiological reactions. When the heart is injured or overloaded, a series of abnormal changes will occur in cardiac myocytes and myocardial interstitium, resulting in changes in the shape, size, wall thickness and structure of the heart. At the later stage of ventricular 


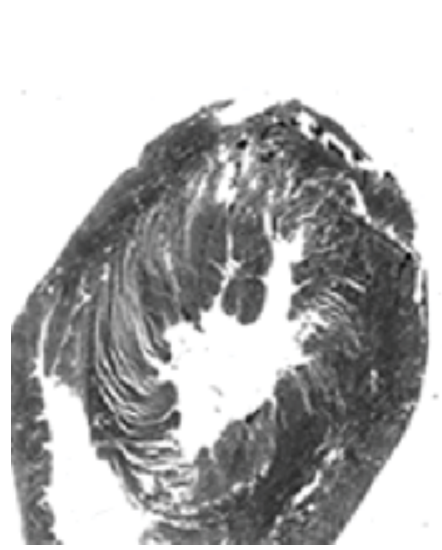

A

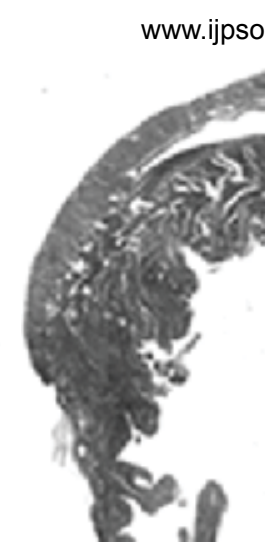

B

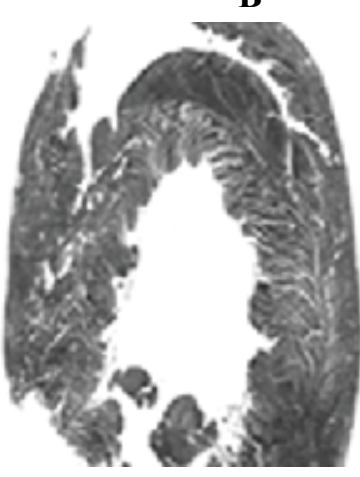

D

Fig. 2: Masson staining results of rat hearts in each group $(400 \mathrm{X})$

A. Blank group, B. control group, C. sham-operated group, D. A1 group and E. A2 group ventricular remodeling after MI, inhibit apoptosis of myocardial cells and improve cardiac function, which is worthy of clinical reference and application. However, the mechanism of ventricular remodeling after MI is complex and related to the mechanism of endocrine system. There are some limitations in this respect, which will be further improved in the future work.

\section{REFERENCES}

1. Xiao K, Bin K, Xing W. Additive effects of atorvastatin combined with sitagliptin on rats with myocardial infarction: a pilot study. Arch Med Sci 2017;4:956-61.

2. Yang Y, Zhao J, Zhang J. Regulation of macrophage migration in ischemic mouse hearts via an AKT2/NBA1/SPK1 pathway. Oncotarget 2017;8(70):115345-59.

3. Jie R, Wei L, Guang-Cai L. Atorvastatin Attenuates Myocardial Hypertrophy Induced by Chronic Intermittent Hypoxia In Vitro Partly through miR-31/PKC $\varepsilon$ Pathway. Current Medical Science 2018;38(3):405-12.

4. Lu C, Jia H, Wang Z. High-dose atorvastatin reduces the risk of cardiovascular events in patients with percutaneous coronary intervention Authors and affiliations. Oncotarget 2017;8(41):70356-65.

5. Yu Y, Yin G, Bao S. Kinetic alterations of collagen and elastic fibres and their association with cardiac function in acute myocardial infarction. Mol Med Rep 2018;17(3):3519-26.

6. Shehata M, Samir A, Dardiri M. Prognostic impact of intensive statin therapy on N-terminal pro-BNP level in non-ST-segment elevation acute myocardial infarction patients. J Interv Cardiol 2017;30(6):514.

7. Peng Y, Ou BQ, Li HH. Synergistic Effect of Atorvastatin and 
Folic Acid on Cardiac Function and Ventricular Remodeling in Chronic Heart Failure Patients with Hyperhomocysteinemia. Medical science monitor: Int Med $J$ Exp Clin Res 2018;24:3744-51.

8. Berwanger O, Pedro GM, de Barros e Silva, Barbosa RR. Atorvastatin for high-risk statin-naïve patients undergoing noncardiac surgery: The Lowering the Risk of Operative Complications Using Atorvastatin Loading Dose (LOAD) randomized trial. Am Heart J 2017;184:88-96.
This is an open access article distributed under the terms of the Creative Commons Attribution-NonCommercial-ShareAlike 3.0 License, which allows others to remix, tweak, and build upon the work non-commercially, as long as the author is credited and the new creations are licensed under the identical terms

This article was originally published in Special
issue on "Trends in therapeutic Management of
Various Conditions" Indian J Pharm Sci 2020:82(3) 